\title{
Subxiphoid uniportal video assisted thoracoscopic lobectomy in a pediatric patient
}

\author{
Firas Abu Akar'1, Bisanne Shaqqura1, Nisreen Rumman², Lei Jiang ${ }^{3}$ \\ 'Department of Cardiothoracic surgery, Makassed Charitable Society Hospital, East Jerusalem 97103, Palestine. \\ ${ }^{2}$ Department of pediatrics, Makassed Charitable Society Hospital, East Jerusalem 97103, Palestine. \\ ${ }^{3}$ Department of Thoracic Surgery, Shanghai Pulmonary Hospital, Tongji University School of Medicine, Shanghai 200433, \\ China.
}

Correspondence to: Dr. Firas Abu Akar, Department of Cardiothoracic Surgery, Al-Makassed Charitable Society Hospital, East Jerusalem, Affiliated to AI-Quds University School of Medicine, Jerusalem 97103, Palestine. E-mail: firasabuakar@gmail.com

How to cite this article: Abu Akar F, Shaqqura B, Rumman N, Jiang L. Subxiphoid uniportal video assisted thoracoscopic lobectomy in a pediatric patient. Mini-invasive Surg 2020;4:10. http://dx.doi.org/10.20517/2574-1225.2019.65

Received: 25 Dec 2019 First Decision: 16 Jan 2020 Revised: 17 Jan 2020 Accepted: 3 Feb 2020 Published: 14 Feb 2020

Science Editor: Noriyoshi Sawabata Copy Editor: Jing-Wen Zhang Production Editor: Tian Zhang

\begin{abstract}
Thoracoscopic surgeries have witnessed tremendous and prompt recent development, especially in the field of uniportal video assisted thoracoscopic surgery (VATS) surgery. It is now possible to perform the most complex surgeries through this technique, which is of great benefit to the patient by significantly reducing the level of postoperative pain and complications of surgery. As surgeons gain experience in this field, their confidence and ability to push the limits and develop technologies are increasing. Performing uniportal VATS surgeries in children is a significant challenge for the surgeon due to the limited size of the thoracic cavity and the difficulty of the instrumentation. Here, we report the first case in the literature (as far as we know) of a uniportal Subxiphoid VATS lobectomy in a 2.5-year-old child. In conclusion, Subxiphoid uniportal VATS lobectomy is feasible in pediatric patients and may have some benefits over the intercostal approach.
\end{abstract}

Keywords: Subxiphoid uniportal video assisted thoracoscopic surgery in pediatrics, minimally invasive thoracic surgery in children, VATS lobectomy in pediatrics, pediatric thoracic surgery

\section{INTRODUCTION}

Despite the significant development of adult thoracoscopic surgery in the last two decades, especially the uniportal video assisted thoracoscopic surgery (VATS) technique, which may be performed through the intercostal, subxiphoid, or subcostal approach, the utilization of the uniportal technique in children is still

\footnotetext{
(ब) (1)

(C) The Author(s) 2020. Open Access This article is licensed under a Creative Commons Attribution 4.0 International License (https://creativecommons.org/licenses/by/4.0/), which permits unrestricted use, sharing, adaptation, distribution and reproduction in any medium or format, for any purpose, even commercially, as long as you give appropriate credit to the original author(s) and the source, provide a link to the Creative Commons license, and indicate if changes were made.
}

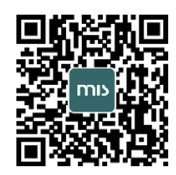




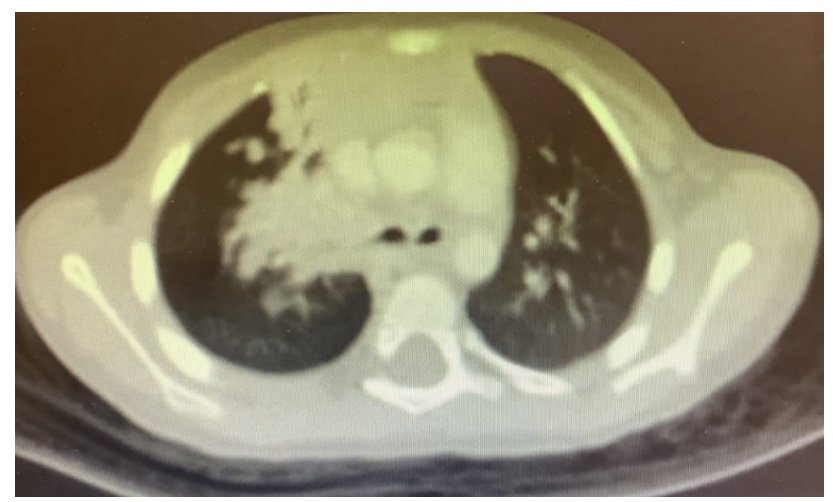

Figure 1. CT scan showing diffuse consolidation and bronchiectasis of the right upper lobe due to severe bronchomalacia

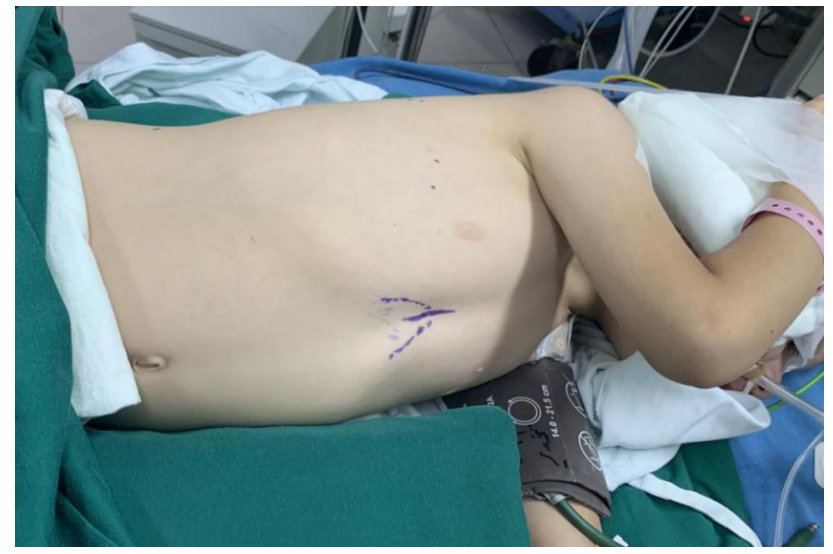

Figure 2. Left decubitus position with the right hand fixed to the right ear to open the axillary space

in its beginning and is rarely performed. The surgical literature contains very few cases, which have been conducted only by experts in this field, and most centers in the world still adopt the traditional methods, either open thoracotomy or the multiportal VATS technique ${ }^{[1,2]}$. After gaining extensive experience in single-port surgery in all its forms in adults and performing it at an advanced level, we started to apply this technique in children as well ${ }^{[3-7]}$. One of the challenges a surgeon may face during a lobectomy in children through the intercostal approach is the small space between the ribs, which may make the instrumentation very difficult and challenging ${ }^{[7]}$. Therefore, we found that it makes sense to perform this type of operation via the subxiphoid approach, which may provide more space for the instrumentation in addition to comfortable angles for the instruments during the dissection of the hilum. In this article, we report the first case of subxiphoid uniportal VATS lobectomy conducted for a 2.5 -year-old child, and we review some of the observations we found during the surgery.

\section{CASE PRESENTATION}

A thirty-month-old male patient suffered from recurrent chest infections since birth, which necessitated several hospital admissions and antibiotics therapy. The chest CT scan showed right upper lobe consolidation and bronchiectasis [Figure 1]. Bronchoscopy was performed to rule out any intrinsic factor or other associated anomalies. The procedure showed a significant narrowing of the right upper lobe bronchus due to severe bronchomalacia. The echocardiogram showed no cardiac abnormalities. The multidisciplinary team forum decided that lobectomy is indicated.

\section{Surgical technique}

The operation was performed under general anesthesia. Isolated right lung ventilation was obtained by advancing an uncuffed single-lumen endotracheal tube to the left main bronchus. The baby was positioned 


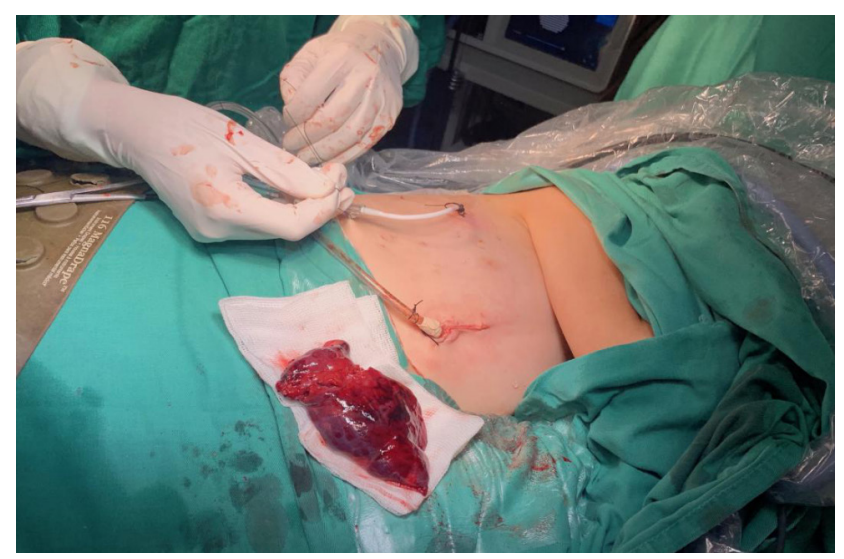

Figure 3. An image showing the chest drainage through the same incision after its closur

in the left decubitus position with two overlapped towel sheets supporting both sides. The right hand was fixed to the right ear to open the axillary space [Figure 2]. A $3-\mathrm{cm}$ incision was made over the xiphoid process [Figure 3]. The subcutaneous tissue was dissected and the insertions of the rectus muscles to both costal arches were divided at the midline. The cartilaginous xiphoid process was excised using surgical scissors. The left pleural space was opened by blunt finger dissection. A wound protector was placed, through which a $30^{\circ} / 5-\mathrm{mm}$ video thoracoscope and all thoracoscopic instruments were introduced into the right pleural cavity.

The right upper lobe was grasped using a lung grasper and then retracted posteriorly and caudally to expose the hilar structures. Specially designed curved tip spatula, harmonic energy device, and fine vascular clamp dissector were used to dissect and encircle the right superior pulmonary vein. Advancing a stapler to divide the vein through the same incision was smooth and more natural than the intercostal approach, and the angles for the staplers were more convenient. The right superior pulmonary vein was stapled using a vascular stapler (Endo GIA ${ }^{\mathrm{TM}}$ Curved Tip Reload with Tri-Staple ${ }^{\mathrm{TM}}$ Technology) [Video 1]. The pulmonary artery was subsequently approached; dissecting and encircling the truncus anterior branch of the pulmonary artery was performed; and the branches were divided after applying two metal clips (5-mm Endo Clip ${ }^{\mathrm{TM}}$ ) using a harmonic scalpel [Video 1]. The left upper lobe bronchus was identified, dissected, and encircled; the vascular stapler (Endo GIA ${ }^{\mathrm{TM}}$ Curved Tip Reload with Tri-Staple ${ }^{\mathrm{TM}}$ Technology) was advanced; and the bronchus was divided. The fissure was completed and divided, including the posterior ascending arterial branch, using a vascular stapler (Endo GIA ${ }^{\mathrm{TM}}$ Reload with Tri-Staple ${ }^{\mathrm{TM}}$ Technology). The resected lobe was extracted out of the thoracic cavity, and the endotracheal tube was withdrawn a few centimeters to check the patency of the lower lobe bronchus with inflation test. Hemostasis was done, a 14 -fr chest drain was inserted through the same incision, and an 8 -fr intercostal microtube was introduced into the pleural space [Figure 3]. The incision was closed in layers, and the patient was extubated and transferred to the pediatrics intensive care unit in a stable condition.

\section{Postoperative course}

The baby was transferred to the pediatric ward $24 \mathrm{~h}$ after the surgery. There were no complications, and the chest drains were removed on the third pos operative day (POD). The patient was discharged from the hospital on the sixth POD in excellent condition [Figure 4].

\section{DISCUSSION}

Since Rodgers and Talbert ${ }^{[8]}$ introduced the thoracoscopic surgery in pediatrics in the 1970s, the topic has not attracted much attention in the field due to some difficulties. Rothenberg is one of the pioneers 


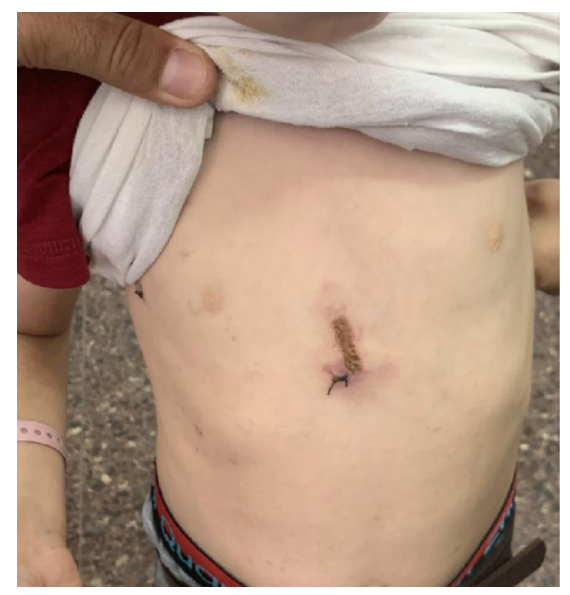

Figure 4. The subxiphoid wound, one week after surgery

who developed the multiportal VATS technique for pediatrics over the last three decades ${ }^{[1,2,9]}$. Besides the cosmetic and early postoperative morbidity concerns, some of the crucial benefits of thoracoscopic surgery in children (which may not affect the adult patients) are decreasing the risk of musculoskeletal deformity (asymmetric chest wall, scoliosis, rib fusion, and winged scapula) that may be noted after posterolateral thoracotomy in younger patients ${ }^{[10-12]}$. In adult patients, a natural progression and evolving of thoracoscopic techniques resulted recently in the evolution of uniportal VATS technique ${ }^{[3-6,13]}$. Mastering the technique of uniportal VATS in adults requires intensive training to pass the learning curve to be applied safely in $\operatorname{adults}^{[6]}$. The subxiphoid approach is a new addition to the uniportal VATS technique. The subxiphoid area is distinguished from the intercostal by being nerveless, which allows avoiding injury to the intercostal nerve during operation ${ }^{[14,15]}$. Besides, the proper angles for the instruments and staplers, which intersect at a $90^{\circ}$ angle with the hilar structures, are more streamlined and comfortable to the operator. However, the subxiphoid approach is not devoid of challenges; the need to compress the pericardium occasionally during surgery and the distance of the work area from the incision requires specialized training and experience. In children, this technique may require additional skills. It could be more challenging to the surgeon for many reasons, including the difficulty of obtaining isolated lung ventilation due to the lack of a double-lumen endotracheal tube in young children, in addition to the lack of appropriate instruments with the curved tip specially designed for uniportal VATS operations. A few reports and studies have recently been published on the utilization of uniportal VATS for anatomical resections in pediatrics ${ }^{[7,16-18]}$. However, the literature does not yet contain a report on subxiphoid uniportal VATS anatomical resection in a pediatric patient. To our knowledge, this is the first case in which an anatomical resection was performed for a patient of this age and weight via subxiphoid uniportal VATS approach.

In conclusion, in expert hands, subxiphoid uniportal VATS lobectomy in pediatrics may be safe and may have benefits to the patient. The surgeon's experience in this type of surgery in adults is crucial before starting to apply it to pediatrics. There is a need to design suitable instruments and staplers for this type of surgery in pediatrics. There is, of course, also a need to run a comparative study with an appropriate cohort of patients before determining the safety and feasibility of this technique in pediatrics.

\section{DECLARATIONS}

\section{Authors' contributions}

Conception and design of the study and performed data analysis and interpretation: Abu Akar F, Shaqqura B Performed data acquistion, as well provided administrative, technical, and material support: Abu Akar F, Jiang L, Rumman N 


\title{
Availability of data and materials
}

The authors declares that all the data that support our findings can be found in our database and archive at Al-Makassed Hospital. Data can be deposited into data repositories or published as supplementary information in the journal.

\section{Financial support and sponsorship}

None.

\section{Conflicts of interest}

All authors declared that there are no conflicts of interest.

\section{Ethical approval and consent to participate}

Ethical approval and consent has been obtained from the patient's parents to publish the article.

\author{
Consent for publication \\ Not applicable.
}

\section{Copyright}

(c) The Author(s) 2020.

\section{REFERENCES}

1. Rothenberg SS. Thoracoscopy in infants and children. Semin Pediatr Surg 1998;7:194-201.

2. Rothenberg SS, Middlesworth W, Kadennhe-Chiweshe A, Aspelund G, Kuenzler K, et al. Two decades of experience with thoracoscopic lobectomy in infants and children: standardizing techniques for advanced thoracoscopic surgery. J Laparoendosc Adv Surg Tech A 2015;25:423-8.

3. Abu Akar F, Gonzalez-Rivas D, Ismail M, Deeb M, Reichenshtein Y, et al. Uniportal video-assisted thoracic surgery: the Middle East experience. J Thorac Dis 2017;9:871-7.

4. Abu Akar FE, Zheng H, Ali G, Zhao X. Bilateral uniportal video-assisted thoracoscopic lung resections. Interact Cardiovasc Thorac Surg 2017;25:1012-3.

5. Bertolaccini L, Batirel H, Brunelli A, Gonzalez-Rivas D, Ismail M, et al. Uniportal video-assisted thoracic surgery lobectomy: a consensus report from the Uniportal VATS Interest Group (UVIG) of the European Society of Thoracic Surgeons (ESTS). Eur J Cardiothorac Surg 2019;56:224-9.

6. Abu Akar F, Gonzalez-Rivas D. Training in an ultra-high-volume center. Video-Assist Thorac Surg 2018;3:17.

7. Shaqqura B, Rumman N, Rivas DG, Abu Akar F. Uniportal video-assisted thoracoscopic lobectomy in a 9-week-old patient. Interact Cardiovasc Thorac Surg 2020;30:327.

8. Rodgers BM, Talbert JL. Thoracoscopy for diagnosis of intrathoracic lesions in children. J Pediatr Surg 1976;11:703-8.

9. Slater BJ, Rothenberg SS. Thoracoscopic management of patent ductus arteriosus and vascular rings in infants and children. J Laparoendosc Adv Surg Tech A 2016;26:66-9.

10. Korovessis P, Papanastasiou D, Dimas A, Karayannis A. Scoliosis by acquired rib fusion after thoracotomy in infancy. Eur Spine J 1993;2:53-5.

11. Vida VL, Tessari C, Fabozzo A, Barzon E, Zucchetta F, et al. The evolution of the right anterolateral thoracotomy technique for correction of atrial septal defects: cosmetic and functional results in prepubescent patients. Ann Thorac Surg 2013;95:242-7.

12. Cherup LL, Siewers RD, Futrell JW. Breast and pectoral muscle maldevelopment after anterolateral and posterolateral thoracotomies in children. Ann Thorac Surg 1986;41:492-7.

13. Gonzalez-Rivas D, de la Torre M, Fernandez R, Garcia J. Video: single-incision video-assisted thoracoscopic right pneumonectomy. Surg Endosc 2012;26:2078-9.

14. Hernandez-Arenas LA, Lin L, Yang Y, Liu M, Guido W, et al. Initial experience in uniportal subxiphoid video-assisted thoracoscopic surgery for major lung resections. Eur J Cardiothorac Surg 2016;50:1060-6.

15. Abu-Akar F, Gonzalez-Rivas D, Yang C, Lin L, Wu L, et al. Subxiphoid uniportal VATS for thymic and combined mediastinal and pulmonary resections - a two-year experience. Semin Thorac Cardiovasc Surg 2019;31:614-9.

16. Prasad R, Arthur LG, Timmapuri SJ, Schwartz MZ, Fairbanks TJ, et al. Early experience with single-incision thoracoscopic surgery in the pediatric population. J Laparoendosc Adv Surg Tech A 2011;21:189-92.

17. Park S, Kim ER, Hwang Y, Lee HJ, Park IK, et al. Serial improvement of quality metrics in pediatric thoracoscopic lobectomy for congenital lung malformation: an analysis of learning curve. Surg Endosc 2017;31:3932-8.

18. Gonzalez-Rivas D, Marin JC, Granados JP, Llano JD, Cañas SR, et al. Uniportal video-assisted thoracoscopic right upper sleeve lobectomy and tracheoplasty in a 10-year-old patient. J Thorac Dis 2016;8:E966-9. 